\title{
Yokukan-san: a review of the evidence for use of this Kampo herbal formula in dementia and psychiatric conditions
}

REVIEW

\author{
This article was published in the following Dove Press journal: \\ Neuropsychiatric Disease and Treatment \\ 12 September 2014 \\ Number of times this article has been viewed
}

\author{
Hideki Okamoto 1,2 \\ Masaomi lyo ${ }^{2}$ \\ Keigo Ueda' \\ Cheolsun Han' \\ Yoshiro Hirasaki' \\ Takao Namiki' \\ 'Department of Japanese-Oriental \\ (Kampo) Medicine, ${ }^{2}$ Department of \\ Psychiatry, Chiba University Graduate \\ School of Medicine, Chiba City, Japan
}

Correspondence: Hideki Okamoto Department of Japanese-Oriental (Kampo) Medicine, Chiba University Graduate School of Medicine, I-8-I Inohana, Chuo-ku, Chiba-shi, Chiba 260-8670, Japan

Tel +8I 432262984

Fax +8I 432262985

Email bon@sa2.so-net.ne.jp
Abstract: Japanese traditional herbal medicine (Kampo) has its origins in traditional Chinese medicine (TCM). It was introduced to Japan in the middle of the sixth century and has evolved over the past 1,400 years after combining with Japan's original folk remedies. While it retains some similarities to TCM, Kampo has evolved in Japan, resulting in a system of medicine that has many differences from TCM. Kampo medicine is considered to be very safe; in Japan, Kampo herbal formulas are manufactured by licensed pharmaceutical companies, prescribed by Western-trained medical doctors (usually as a freeze-dried extract), and have quality control standards similar to those of prescription drugs. The present study examined Yokukan-san (Yi-Gan San in TCM), a Kampo formula that has been used empirically in Japan for more than 400 years. Accumulating clinical trials have demonstrated Yokukansan's efficacy in treating patients with behavioral and psychological symptoms of dementia, which has resulted in the Japanese Society of Neurology listing it in the Japanese Guidelines for the Management of Dementia 2010. Efficacy in other diseases and conditions, such as sleep disorders, tardive dyskinesia, aggression, and impulsivity has also been reported. This article reviews both clinical and basic studies of Yokukan-san, with the goal of clarifying its clinical indications.

Keywords: Yokukan-san-ka-chimpi-hange, Japanese traditional medicine, Asian medicine, dementia with Lewy bodies (DLB), Alzheimer's disease, BPSD, Parkinson's disease

\section{Introduction}

Yokukan-san (YKS; also written as Yokukansan or Yoku-kan-san in Japanese) is a Japanese traditional medicine (Kampo) formula derived from Yi-Gan San in traditional Chinese medicine (TCM). YKS has attracted attention in the field of psychiatry due to reports of its efficacy in patients with dementia. ${ }^{1-14}$ In addition, therapeutic effects of YKS have been reported in the treatment of sleep disorders, ${ }^{15,16}$ tardive dyskinesia, ${ }^{17}$ borderline personality disorder, ${ }^{18}$ and schizophrenia-related diseases. ${ }^{19,20}$ The claim of YKS's effectiveness for such a wide variety of indications may produce skepticism toward Kampo medicine and possibly weaken its reputation, especially if YKS is ineffective or harmful because of improper usage. YKS has been approved by the Ministry of Health, Labor, and Welfare in Japan as a covered prescription under the National Health Insurance. This review of YKS provides a general introduction to Kampo medicine, describes the Kampo philosophy, explains the empirical use of YKS based on Kampo diagnosis, and reviews both clinical and basic studies of YKS, with the goal of clarifying the clinical indications of this herbal formula. 


\section{Kampo medicine Overview}

Kampo medicine originated in China and has its roots in TCM. It was introduced to Japan in the middle of the sixth century and has evolved over the past 1,400 years. ${ }^{21,22}$ While it retains some similarities to TCM, Kampo has evolved independently in Japan, resulting in a system of medicine that has many differences from TCM. ${ }^{22-24}$ After its introduction from China, Kampo was combined with Japan's original folk remedies and was modified by the introduction of formulas and herbs that had not been used in TCM. ${ }^{25}$ Kampo medicine was the main medical treatment in Japan until Western medicine was introduced from Europe several hundred years ago. ${ }^{21-24}$

\section{Kampo herbal formulas in Japan}

Although some Kampo herbal formulas are based on classical TCM formulas, the Kampo formulas have been standardized in Japan and use fixed combinations of herbs. ${ }^{21,22,24}$ Kampo herbal formulas are regulated in the same manner as pharmaceutical preparations, and are approved and manufactured with standards that are equivalent to those of conventional pharmaceutical medicines. Both manufacturers and the government are involved in extensive monitoring of agricultural and manufacturing processes, as well as in post-marketing surveillance, in order to ensure the quality and safety of these products. The result of this emphasis on standardization is that while a single Kampo herbal formula might be produced by various different manufacturers, it will be subject to the same quality control standards. This high degree of quality and standardization does not necessarily apply if the formula is made outside of Japan. ${ }^{21,22,24}$

Kampo is integrated into Japan's national health care system, and all citizens therefore have access to governmentapproved Kampo herbal formulas. The Japanese Ministry of Health, Labor, and Welfare has approved 212 Kampo formulas for clinical use, which means that these formulas are also approved for reimbursement under the National Health Insurance program. Of these 212 Kampo formulas, 148 have granule-type (freeze-dried herbal extract) prescriptions and are manufactured by several licensed pharmaceutical companies in Japan. The daily dose is usually in the range of 4.5-27 (most frequently 6-7.5) g of granules, which are generally divided into two or three doses per day. These prescription Kampo formulas are considered to be much easier to take because of the freeze-dried granules, and safer because of the standardization and quality control procedures than decoctions prepared by patients themselves. ${ }^{22}$
Most physicians in Japan prescribe at least some granule-type Kampo medicines. ${ }^{21,22,24}$

Recently, Kampo medicine has received renewed attention because it provides a valid alternative to treating symptoms refractory to conventional medicine. There has also been an emphasis on evaluating Kampo formulas using modern techniques to evaluate their mechanisms of action and to examine their efficacy in the context of Western models of disease and diagnostic criteria. ${ }^{24}$

\section{Differences between Kampo and conventional Western medicine}

Kampo medicine has a diagnostic scheme that is completely different from that of conventional Western medicine. The prescription of a specific formula is usually based on both the patient's subjective symptoms and objective signs using a Kampo-specific diagnostic examination. This diagnostic approach is discussed in more detail below. Because Kampo prescriptions are usually comprised of multiple herbs, all of which can contain large numbers of active constituents, there is a need to elucidate the clinical effects of these formulas. ${ }^{21-24}$

Within Kampo medicine, a single herbal formula prescription is often used to treat a wide variety of symptoms (a practice called "Ibyodochi" in Kampo). One good example of Ibyodochi is YKS, a single prescription that is reported to be effective in treating many different diseases such as dementia, ${ }^{1-14}$ sleep disorders,,${ }^{15,16}$ tardive dyskinesia, ${ }^{17}$ borderline personality disorder, ${ }^{18}$ and schizophrenia-related diseases. ${ }^{19,20}$ On the other hand, different herbal formulas may also be used to treat the same disease (called "Dobyoichi" in Kampo). For example, in cases of influenza, a Kampo formula called Mao-to (TCM: Ma Huang Tang; English: Ephedra Decoction) might be chosen if the patient has a strong, floating (ie, easily felt), tensed pulse, and shivering followed by a high fever (symptoms suggestive of a vigorous immune response), while a formula called Mao-bushisaishin-to (TCM: Ma Huang Fu Zi Xi Xin Tang; English: Ephedra, Aconite, and Manchurian Wild Ginger Decoction) might be prescribed if the patient has a low-grade fever together with a weak and sunk pulse (symptoms suggestive of a weak or less vigorous response), even though both sets of symptoms are caused by the same influenza virus. ${ }^{22,23}$ Both Ibyodochi and Dobyoichi are often used in the clinical practice of Kampo because Kampo medicine uses a completely different paradigm from conventional diagnostics. The specific prescription is chosen after considering the unique tendencies and reactions to disease of the patient based on 
Kampo philosophies, which focus on enhancing the body's resistance to disease by improving self-controlled systems and correcting any physical or mental imbalance. ${ }^{22,23}$

Another important distinction is that Kampo does not distinguish between psychiatric and physical symptoms because it regards each human being as a complete and self-controlled unit in which mind and body affect each other. In the Kampo approach, most diseases are considered to be psychosomatic in origin and most herbs are believed to affect both the psyche and the soma equally. Therefore, Kampo treatment is focused on the patient as a whole rather than on the disease. This patient-centered approach allows Kampo to be applied even when the etiology of the condition in question is unknown or when the patient's symptoms defy easy classification within Western medicine. For these and other reasons, Kampo can be a valid and useful approach to both physical and mental diseases that are refractory to conventional Western medicine. ${ }^{21-24}$

\section{Kampo diagnosis}

Kampo diagnosis is made based on Kampo-specific philosophies of health and disease, irrespective of conventional Western diagnosis, as mentioned previously. Some of these philosophies derive from TCM, such as the In-Yo (Chinese: Ying-Yang) theory, the Five Phases Theory, and the Six Stages of Disease. Others evolved independently of TCM and are Kampo specific, such as the Ki-ketsu-sui (Qi-BloodWater) theory, which developed in Japan, is based primarily on abdominal diagnosis, and is a widely used method of choosing herbal formula prescriptions in Japan. This abdominal diagnosis method has the advantages of being a standardized method of examination and of evoking pressure pains on palpation at routine abdominal points that are both objective and reproducible, even among doctors without knowledge of Kampo medicine. Pulse diagnosis can also be used. In TCM, pulse diagnosis tends to be based on the sense impression obtained by each doctor based on his or her training or experience; however, it has been simplified and generalized in Japan, so that even primary care doctors without knowledge of Kampo medicine can utilize this Kampo diagnostic technique..$^{21-23}$

Kampo medicine often shows remarkable effectiveness when used in individual cases with the distinctive features that meet the criteria, based on Kampo diagnosis, for choosing a specific Kampo prescription. For example, YKS is appropriate under the following conditions:

- Impatience or frequent anger, irritability, muscular tics or convulsions, and/or blepharospasm.
- According to pulse diagnosis, a tight overall pulse quality, and when the pulse is palpated at three contiguous positions overlying the left and right radial arteries, the relative force of the pulse at the middle position is weak on the left and perhaps also on the right. This pulse pattern indicates liver dysfunction in the Five Phases Theory.

- According to abdominal diagnosis, abdominal tension should not be weak, the rectus abdominis muscle is often strained, and there is often tenderness or discomfort in the hypochondrium on palpation and pulsation at the upper navel region.

\section{Empirical use of YKS}

YKS consists of seven herbs (Table 1). This formula dates back to at least 1555, where it was listed in a Chinese medical book "Bao Ying Cuo Yao". The name of YKS originally meant "medicine suppressing the liver". The original Chinese description states in "Bao Ying Cuo Yao" that YKS:

\begin{abstract}
...treats spasm, feverishness, clenching, terrified/simmering mental state, or intolerance to heat and cold caused by pseudo-hyperfunction of the liver and expectoration of sputum, abdominal fullness, loss of appetite, and sleep disorders caused by dysfunction of the spleen, which has a restraining relationship with the liver and is consequently damaged by the liver's pseudo-hyperfunction.
\end{abstract}

In the Five Phases Theory, the liver is considered to be the organ that stabilizes mental activities and ameliorates involuntary muscle movements. Thus, the liver often produces anger and/or involuntary muscle movement when its regular function is impaired. However, most Kampo practitioners do not practice based solely on the Five Phases Theory. That is, they do not use all Kampo-specific diagnostics, including detailed pulse diagnosis, and Kampo prescriptions are not chosen based on strict adherence to Kampo-specific philosophy. Instead, YKS is chosen based on subjective symptoms, often in combination with Kampo abdominal diagnosis.

YKS has been used empirically as a treatment for insomnia, irritability, impulsivity, aggression, blepharospasm

Table I Components of Yokukan-san

\begin{tabular}{ll}
\hline Constituent herbs & Weight (g) \\
\hline Japanese Angelica root (当帰) & 3.0 \\
Atractylodes lancea rhizome (蒼术) & 4.0 \\
Bupleurum root (柴胡) & 2.0 \\
Poria sclerotium (获苓) & 4.0 \\
Glycyrrhiza root (甘草) & 1.5 \\
Cnidium rhizome (川芎) & 3.0 \\
Uncaria hook (釣藤鈎) & 3.0 \\
\hline
\end{tabular}


(abnormal contraction or twitch of the eyelids), tremor or fasciculation of the facial muscles, convulsions, and similar symptoms. It also has a reputation for being effective in the treatment of screaming attacks, sleep terrors, and hypnic myoclonia, especially in infants. ${ }^{22,23}$ YKS is considered to have some effects on the excitability of nerves. A famous Kampo medicine doctor, Dotaku Meguro (1739-1798), wrote in his book "San'ei-kan-ryochi-zatsuwa", "YKS has some efficacy if the patient feels anger", by which he meant to indicate that YKS could be chosen if this subjective symptom was present and could be expected to exert its efficacy without the need for considering more extensive abdominal and/or tongue diagnosis. Its utility in this specific circumstance might be one of the reasons why YKS can be widely used and still show effectiveness.

\section{Case reports}

The first report of the clinical efficacy of YKS appearing in a Western medical journal was a case report in 2005 written by the authors' group, in which YKS was found to be effective in the treatment of tinnitus, headache, and insomnia in the case of an undifferentiated somatoform disorder. ${ }^{26}$ This report demonstrated the clinical efficacy of YKS in treating both physical and mental symptoms. Since then, other case reports have also described therapeutic effects of YKS. These are summarized in Table 2 .

In one of these other case reports, YKS improved psychosis and sleep disturbance in a case of dementia with Lewy bodies (DLB). ${ }^{1}$ In a case of an 85-year-old man with dementia that developed after traumatic brain injury, YKS markedly reduced aggressive and impulsive behavior that was refractory to conventional neuroleptics, and did this without producing any adverse effects. ${ }^{27}$ In non-dementia diseases, YKS has been reported to reduce visual hallucinations due to vision loss in patients with Charles Bonnet syndrome. ${ }^{28}$ There are also reports on the successful treatment of sleep disorders (three cases of rapid eye movement sleep behavior disorder ${ }^{15}$ and three cases of restless leg syndrome), ${ }^{16}$ post-stroke emotional incontinence (uncontrollable episodes of crying or laughing in two cases), ${ }^{29}$ and nocturnal eating/drinking syndrome accompanied by restless leg syndrome and schizophrenia in one case. ${ }^{30}$ YKS is thus widely used for a variety of diseases in Japan. Furthermore, the authors' group recently reported that the addition of Orengedoku-to, another Kampo formula, to YKS was markedly effective in three cases (Alzheimer-type dementia, tardive dystonia, and intermittent explosive disorder) and that this augmentation with Orengedoku-to is worth trying in case in which the efficacy of YKS is limited. ${ }^{31}$

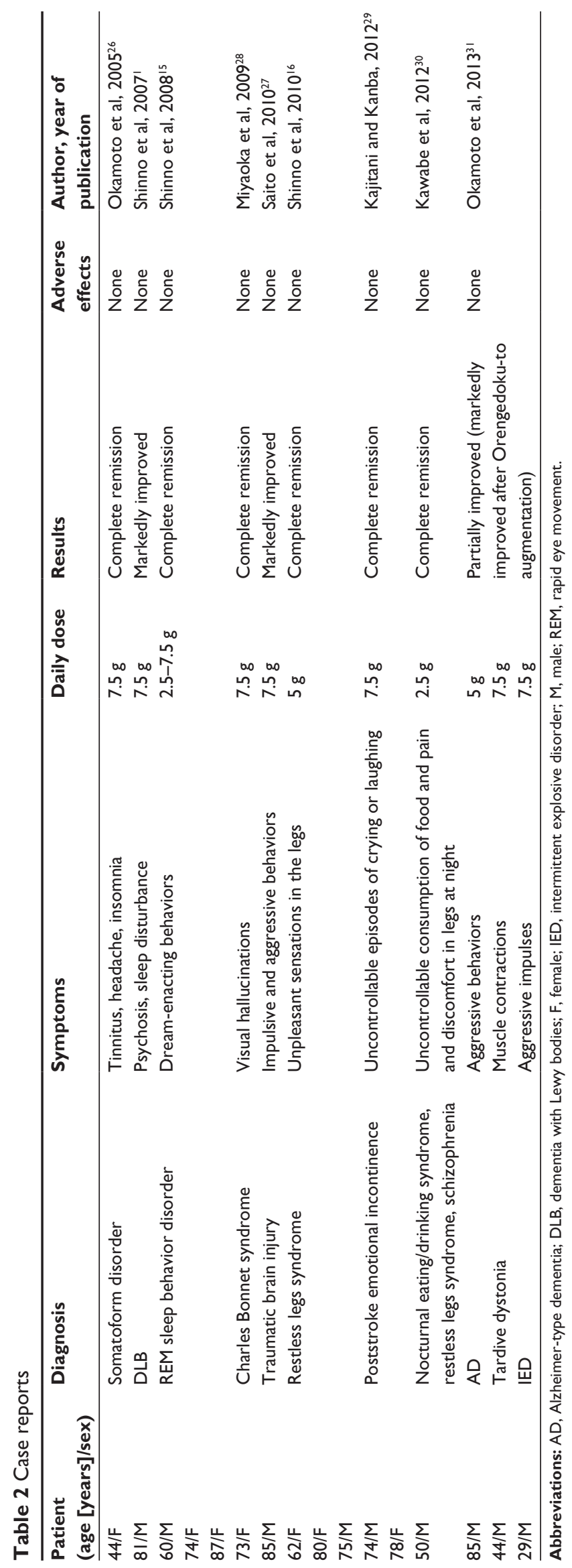


In these cases, YKS has been reported to be effective in the treatment of not only mental but also physical symptoms such as tinnitus, headache, and restless leg syndrome, which is consistent with the notion that Kampo medicine is useful for harmonizing mind and body.

\section{Controlled human studies Studies on dementia}

Although there have been many controlled studies, to the best of the authors' knowledge, there have been no placebocontrolled studies of YKS. Part of the reason for this is that, due to the unique flavor and odor of YKS, it is difficult to prepare a suitable placebo. The first controlled study of YKS (a randomized, observer-blind, multicenter, controlled trial) was performed in early $2005 .^{2}$ In that study, 4 weeks of treatment with YKS significantly improved scores on both the Neuropsychiatric Inventory (NPI) for the assessment of behavioral and psychological symptoms of dementia (BPSD) and the Barthel Index for activities of daily living (ADL) in 52 patients with mild to severe dementia. In this study, MiniMental State Examination results were unchanged. Also of note, extrapyramidal symptoms were not observed with YKS, demonstrating that YKS has a different therapeutic mechanism from that of antipsychotic agents that improve BPSD and ADL without impairing cognitive function or causing extrapyramidal symptoms. This report marked the beginning of the research interest in YKS for the treatment of dementia. Since that time, there have been many other controlled studies on YKS (Table 3). The same group that conducted the original controlled trial in dementia subsequently reported the efficacy of YKS in treating cholinesterase inhibitor-resistant visual hallucinations and neuropsychiatric symptoms of DLB. ${ }^{3}$ Since that time (late 2005), YKS has become the second-line treatment for DLB in Japan. The efficacy of YKS in DLB was further validated in a larger population (54 patients) by the same group in $2012 .{ }^{11}$

In 2009, a randomized crossover study involving 103 dementia patients was carried out and further supported the clinical efficacy of YKS in patients with BPSD. ${ }^{5}$ Another report found that 4 weeks of YKS administration significantly increased total sleep time, sleep efficiency, and stage II sleep, and decreased the numbers of arousals and periodic limb movements as well as ameliorating psychiatric symptoms in BPSD. ${ }^{4}$ This suggests that part of the efficacy of YKS in such cases might be secondary to its effects on sleep.

Three open-label studies have also supported the effectiveness of YKS in people with BPSD and Alzheimer-type dementia. ${ }^{6-8}$ YKS treatment for 4 weeks has been reported to be effective for treating BPSD in patients with Parkinson's disease and those with Parkinson's disease with dementia. Significant improvements in behavioral and psychological symptoms, particularly in the incidence and duration of hallucinations, were observed in most Parkinson's disease and Parkinson's disease with dementia patients; however, no significant changes were observed in laboratory test results, cognitive function, ADL, or parkinsonism. ${ }^{9}$ Four weeks of YKS treatment significantly improved NPI scores and the Stereotypy Rating Inventory in 20 patients with frontotemporal dementia ${ }^{10}$ and NPI scores in 13 patients with vascular dementia, ${ }^{12}$ suggesting that YKS alleviates the behavioral symptoms of frontotemporal and vascular dementia as well as other types of dementia.

YKS has also been found to exert significant therapeutic effects without severe adverse effects on the behavioral symptoms of dementia among chronic renal failure patients receiving hemodialysis three times per week. ${ }^{13}$ To the best of the authors' knowledge, the most recent controlled clinical trial was carried out in 2013 in a well-structured study, in which patients were randomized by the trial investigator to preserve rater blinding to a flexible oral dosing regimen of either YKS, risperidone, or fluvoxamine, and demonstrated that YKS improved NPI scores without causing extrapyramidal symptoms and with no significant between-group differences. $^{14}$

There is only one systematic review of YKS's effectiveness on dementia among four studies ${ }^{2,5,6,8}$ published in $2013 .{ }^{32}$ The meta-analysis in this review concluded that YKS was superior to usual care (control) in the reduction of total NPI scores $(P=0.0009)$ and ADL scores $(P=0.04)$, and more efficacious especially in reducing scores on some NPI subscales (delusions, hallucinations, and agitation/aggression) than usual care $(P<0.00001-0.0009)$.

\section{Controlled studies on other diseases}

YKS's therapeutic effects on diseases other than dementia have also been reported since 2008 (Table 4). An open-label study reported the efficacy of YKS on visual hallucinations due to vision loss in patients with Charles Bonnet syndrome in 2011. ${ }^{33}$ Three reports have suggested the potential for use in persons with schizophrenia-related diseases..$^{17,19,20}$ Four weeks of adjunctive therapy with YKS for treatment-resistant schizophrenia has been found to decrease Positive and Negative Syndrome Scale scores on the schizophrenia subscale without changing Drug-Induced Extrapyramidal Symptom Scale total scores. ${ }^{19}$ Similarly, 4 weeks of adjunctive therapy with YKS for very late-onset schizophrenia-like psychosis 


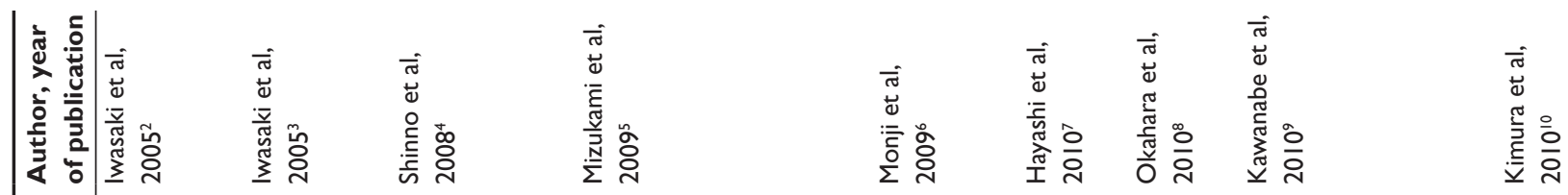
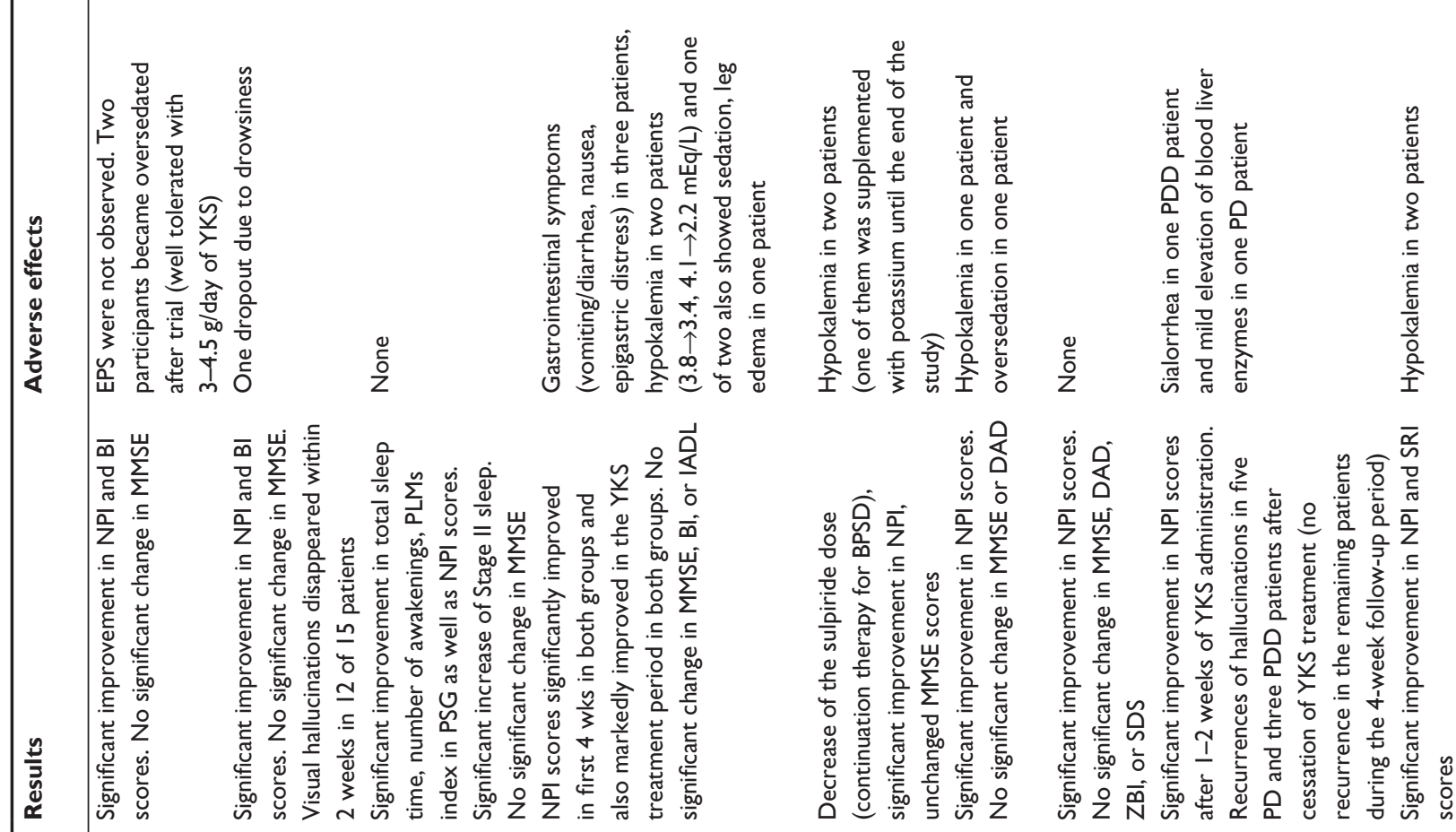

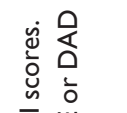

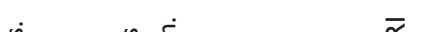

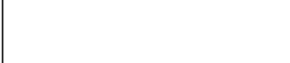

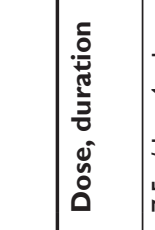
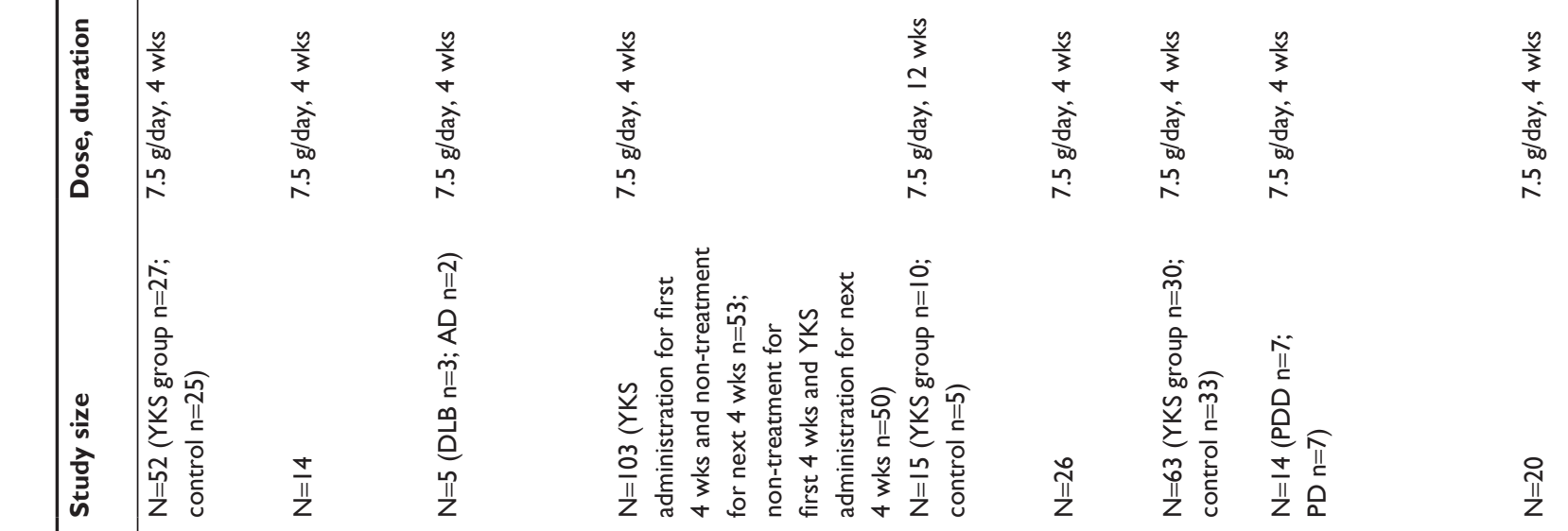

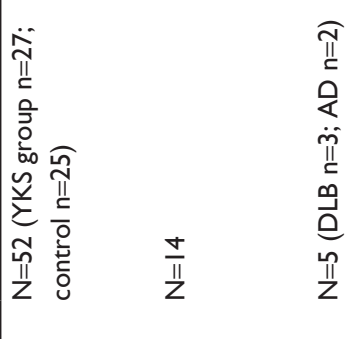
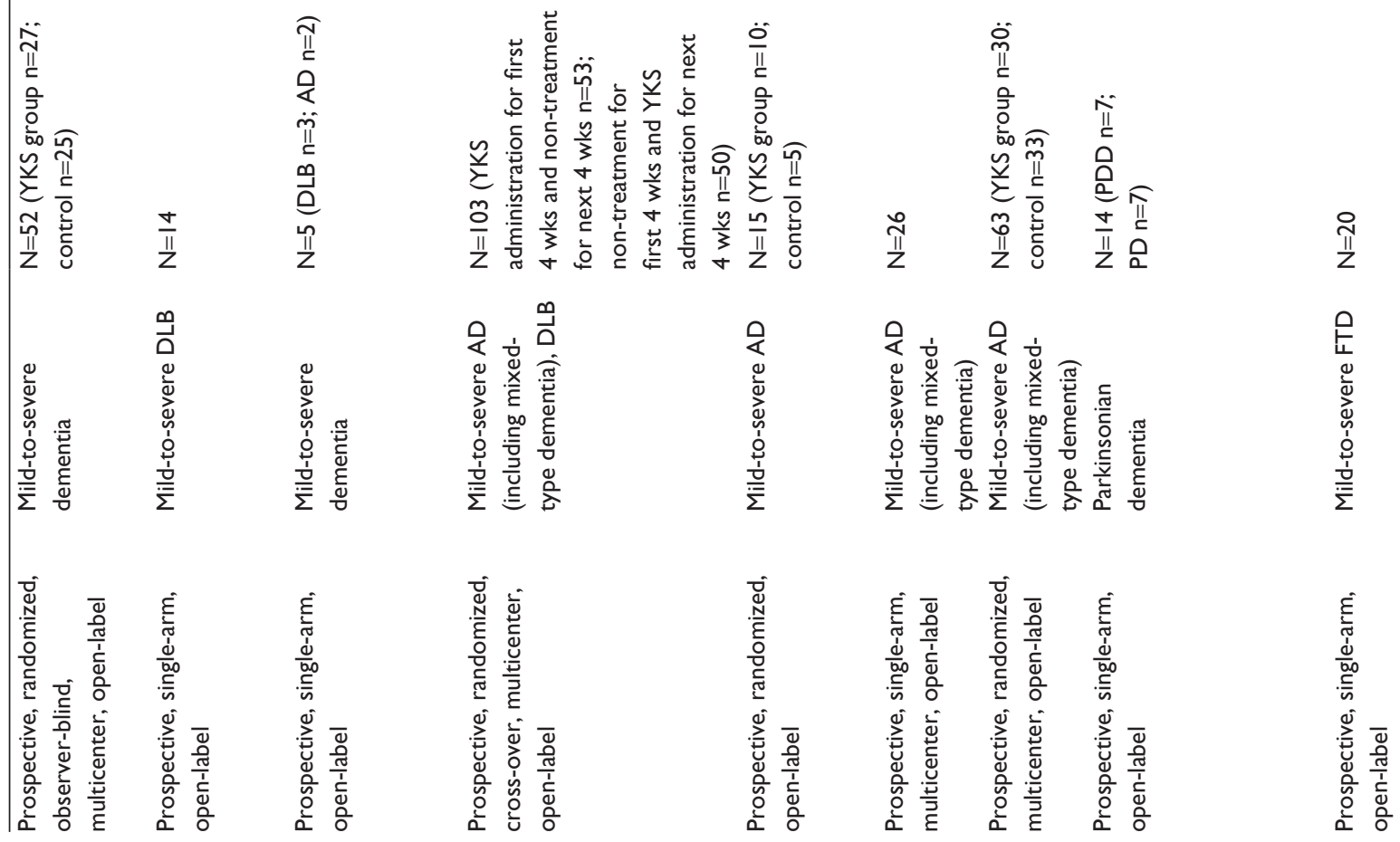


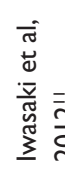

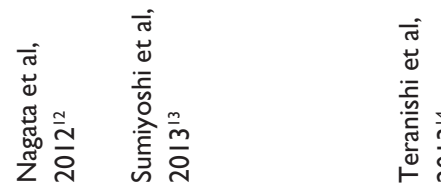

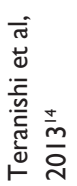
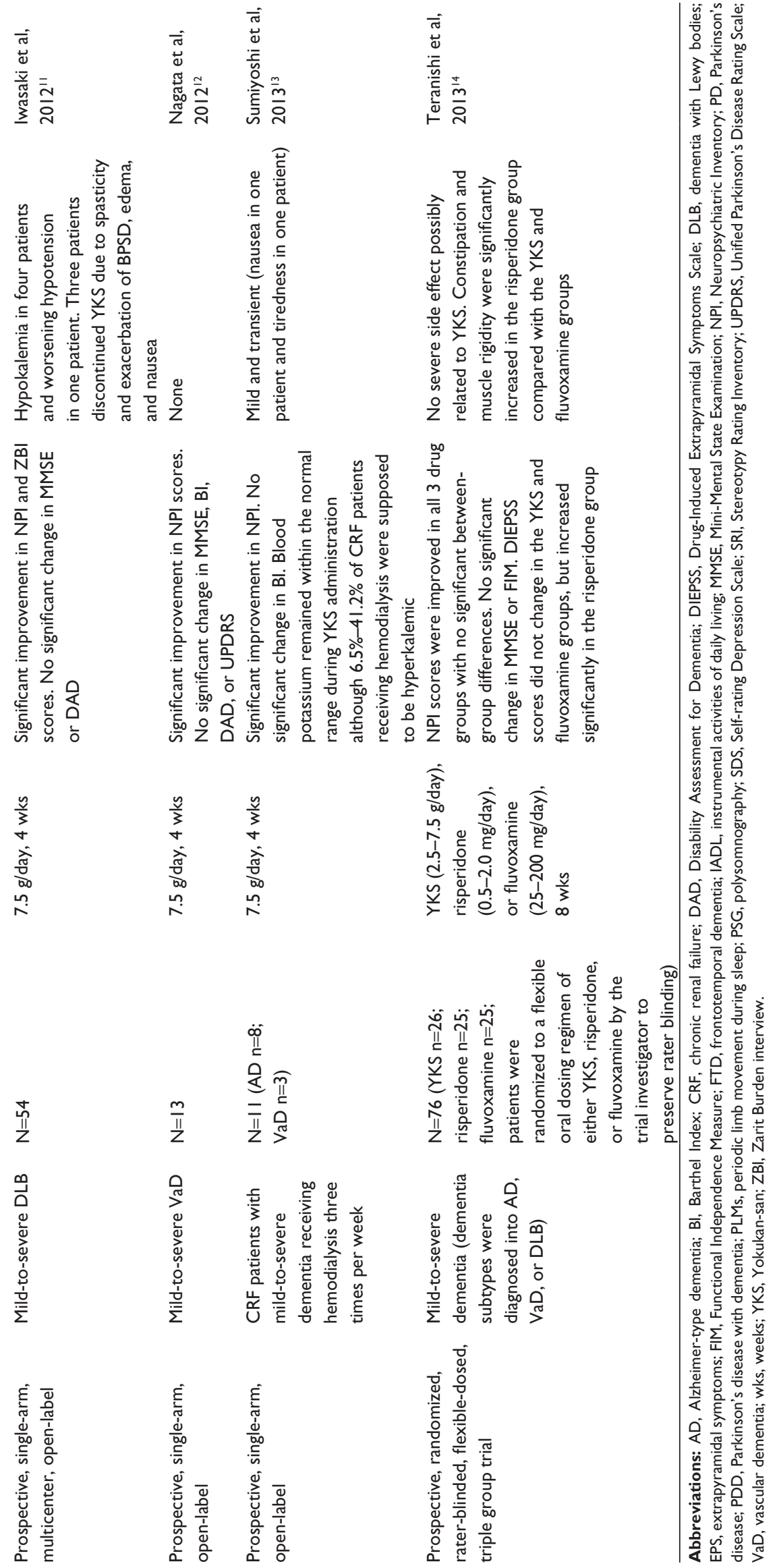


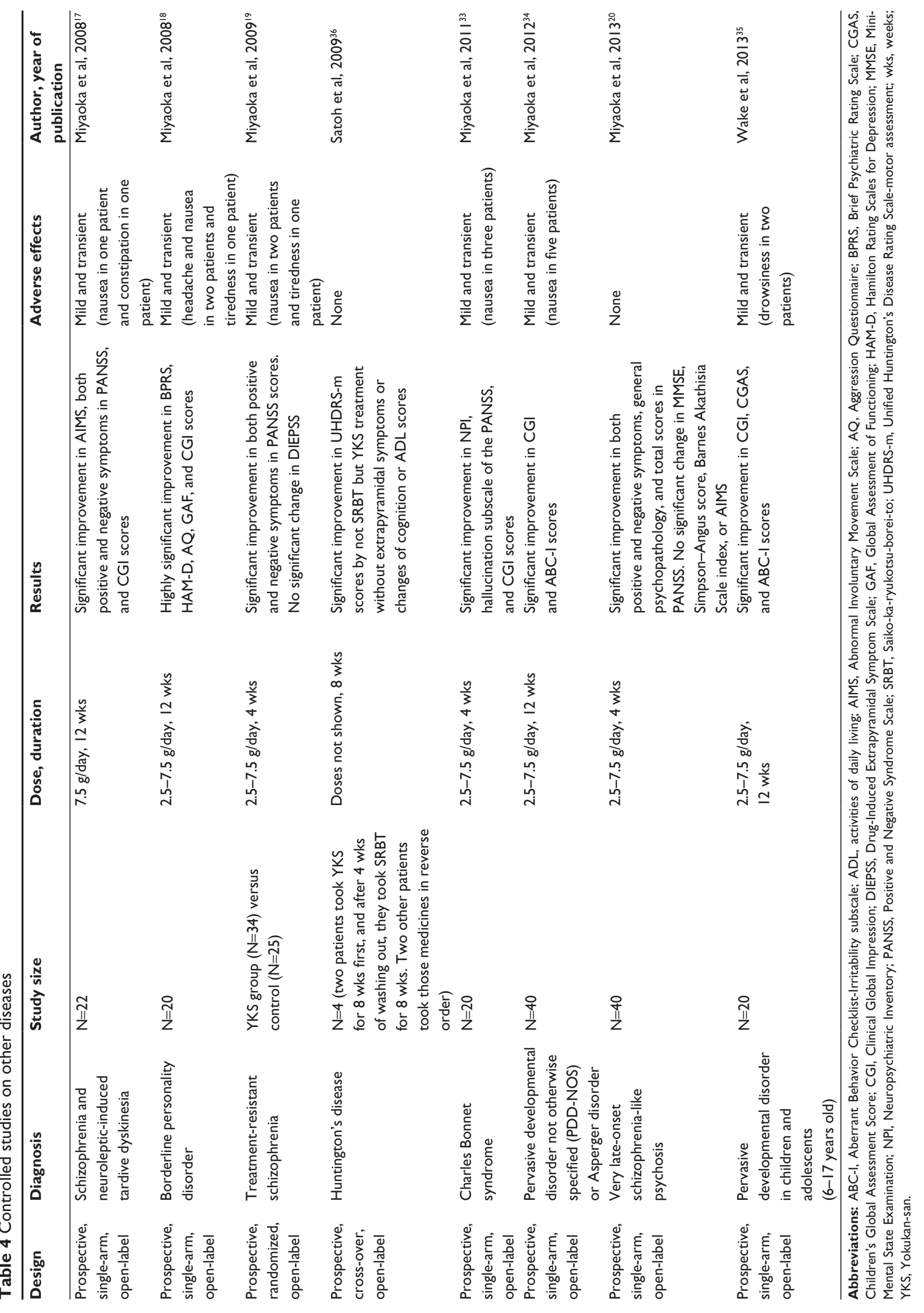


significantly improved Positive and Negative Syndrome Scale, Brief Psychiatric Rating Scale, and Clinical Global Impressions scores without impairing cognitive function or aggravating extrapyramidal symptoms. ${ }^{20}$ Twenty-two patients with schizophrenia who had neuroleptic-induced tardive dyskinesia were given YKS for 12 weeks in an open-label study. ${ }^{17}$ Intriguingly, there was a statistically significant improvement in both tardive dyskinesia and psychotic symptoms. These three studies suggest that YKS may be a safe adjunctive therapy in persons with refractory schizophrenia or schizophrenia-like psychosis with vulnerability to extrapyramidal adverse effects.

One study suggests benefits in patients with borderline personality disorder. ${ }^{18}$ Twenty female outpatients diagnosed with this psychiatric condition completed a 12-week openlabel study with YKS. There was a statistically significant reduction on both self-rated and clinician-rated scales for low mood, impulsivity, and aggression. Furthermore, two studies reported that YKS improved Aberrant Behavior ChecklistIrritability subscale and Clinical Global Impressions scores in subjects with pervasive developmental disorders and Asperger disorder. ${ }^{34,35}$

Benefits for neurological symptoms in neurodegenerative disease have also been reported. As mentioned earlier, within Kampo medicine, involuntary movements are one of the criteria for considering YKS. Four Huntington's disease patients showed a significant decrease in their Unified Huntington's Disease Rating Scale-motor assessment scores following 8 weeks of YKS treatment; in this study there were no extrapyramidal symptoms or changes in cognition or ADL. ${ }^{36}$

In summary, various controlled studies have demonstrated the effectiveness of YKS not only on emotion-specific symptoms and psychotic symptoms but also on neurological symptoms. This is consistent with published case reports showing its effectiveness in individuals with both mental and physical symptoms, and with the traditional use of this Kampo formula.

\section{Animal studies}

A variety of animal studies have been done to support the clinical efficacy of YKS and elucidate its therapeutic mechanisms, which are summarized in Table 5.

\section{Antidepressant-like and pain-relieving effects}

To test the antidepressant-like and pain-relieving effects of YKS, a study was designed to assess behavioral and mood effects under circumstances intended to cause behavioral despair. ${ }^{37}$ YKS was administered for 14 consecutive days in the drinking water of mice. The mice were then placed for 15 minutes in a water tank from which there was no escape containing a water wheel. In this model, the number of wheel rotations counted as escape attempts. YKS administration had a biphasic effect, markedly increasing the number of wheel rotations at lower doses ( 60 and $150 \mathrm{mg} / \mathrm{kg} / \mathrm{day}$ ) but decreasing the number at the highest dose $(300 \mathrm{mg} / \mathrm{kg} /$ day $)$. This study suggests the possibility that YKS has antidepressantlike effects at some doses and counterproductive effects at other doses. In the same paper, YKS was reported to reduce the number of acetic acid-induced writhing responses and have an antinociceptive function.

YKS is sometimes effective in clinical practice for ameliorating neuropathic pain symptoms and another paper reports on YKS's pain-relieving effect, validating such clinical efficacy in a rat chronic constriction injury model ${ }^{38} \mathrm{YKS}$ significantly inhibited mechanical and cold allodynia in the von Frey hair or acetone test, respectively, while amitriptyline, a tricyclic antidepressant, demonstrated moderate, but not significant, antiallodynic effects in the same tests. YKS significantly inhibited the cerebrospinal fluid dialysate level of glutamate, which had been increased by stimulation with a brush or acetone, and the glutamate transporter inhibitors DL-threo- $\beta$-hydroxyaspartate and dihydrokainate decreased YKS-induced antiallodynic actions in chronic constriction injury rats, suggesting that the antiallodynic effects of YKS are related to glutamatergic neurotransmission via the activation of glutamate transporters.

\section{Anti-aggressive properties}

Animal studies have also revealed anti-aggressive properties of YKS. This is consistent with both traditional uses and results from human studies. YKS has been found to ameliorate aggressiveness induced by social isolation in rats, ${ }^{39}$ by zinc deficiency in young mice, ${ }^{40}$ by social isolation in zinc-deficient and pair-fed mice, ${ }^{41}$ by the intravenous injection of para-chloroamphetamine in rats, ${ }^{42}$ and by the intracerebroventricular injection of amyloid $\beta$ protein in mice, which is a model for Alzheimer's disease. ${ }^{43}$ Additionally, YKS $(300 \mathrm{mg} / \mathrm{kg})$ inhibited methamphetamineinduced hyperlocomotion, while YKS at the same dose had no effect on hyperlocomotion induced by MK-801, an $\mathrm{N}$-methyl-D-aspartate receptor antagonist, suggesting that the psychopharmacologic effects of YKS might be mediated by inhibiting the activity of the dopaminergic system. ${ }^{39}$ Another paper discusses the possibility that the anti-aggressive effect of YKS may be related to the glutamatergic system. ${ }^{41}$ 
Table 5 Animal studies

\section{Antidepressant-like effects}

YKS increased the number of wheel rotations in a water tank, counted as escape attempts, at lower doses (60 and I50 mg/kg/day) and decreased the number at the highest dose $(300 \mathrm{mg} / \mathrm{kg} / \text { day })^{37}$

\section{Pain-relieving effects}

- YKS reduced the number of acetic acid-induced writhing responses ${ }^{37}$

- YKS inhibited mechanical and cold allodynia in the von Frey hair or acetone test, respectively ${ }^{38}$

\section{Anti-aggressive properties}

YKS ameliorated aggressiveness induced by:

- Social isolation ${ }^{39}$

- Zinc deficiency ${ }^{40}$

- Social isolation in zinc-deficient and pair-fed mice ${ }^{41}$

- The intravenous injection of para-Chloroamphetamine ${ }^{42}$

- Intracerebroventricular injection of amyloid beta protein ${ }^{43}$

\section{Anxiolytic effects}

- Repeated administration of YKS decreased anxiety-like behavior in an open-field test and an elevated plus-maze test in a dose-dependent manner ${ }^{45}$

- YKS's anxiolytic effect was inhibited by flumazenil, a benzodiazepine receptor antagonist ${ }^{46}$

- Three months of YKS administration improved age-related anxiety in F344/N aged rats ${ }^{47}$

- Repeated administration of YKS suppressed rats' freezing behavior on re-exposure to the box where they had experienced footshock in a contextual fear conditioning test and increased the time spent in open arms in rats subjected to footshock stress compared to control rats ${ }^{48}$

\section{Efficacy of YKS in dementia models of Alzheimer's disease}

- Treatment with YKS for 2 months reduced the increased number of entries and the time spent in open arms in the elevated plus-maze test, treatment for 5 months shortened the time until reaching the platform in the Morris water maze test, and treatment for 9 months suppressed the increase in locomotion in the open-field test in the Tg2576 mice expressing the human form of the APP695SWE ${ }^{49}$

- YKS ameliorated spatial memory impairment in an early-phase Alzheimer's disease rat model ${ }^{51}$

- YKS improved learning and memory impairments in olfactory bulbectomized mice ${ }^{52}$

Studies using animal models of other dementia types

YKS ameliorated:

- Memory disturbance as well as BPSD-like behavior such as anxiety-like behavior, increases in aggressive behaviors, and decreases in social behaviors in thiamine-deficient rats $^{53}$

- Age-related impairments of working memory and reversal learning in aged (24-month-old) rats ${ }^{55}$

- Anxiety-like behaviors in rats subjected to repeated cerebral ischemia ${ }^{56}$

Studies on other clinical efficacies of YKS

- YKS ameliorated vacuous chewing movement in rats, an index for tardive dyskinesia, caused by haloperidol decanoate ${ }^{57}$

- YKS had a reversal effect on the decrease in pentobarbital sleep by social isolation without affecting pentobarbital sleep in group-housed mice ${ }^{58}$

Abbreviations: BPSD, behavioral and psychological symptoms of dementia; YKS, Yokukan-san.

Both MK-801 and aminooxyacetic acid, a $\gamma$-amino butyric acid (GABA) transaminase blocker, attenuated aggressive behavior in zinc-deficient mice but not in pair-fed mice, while YKS (312 mg/kg/day) attenuated aggressive behavior in both groups of mice, suggesting that YKS ameliorates the social isolation-induced aggressive behavior of zinc-deficient and pair-fed mice through action against the glutamatergic neurotransmitter system and other neurotransmitter systems. YKS's ameliorating effect on the isolation-induced aggressive behavior of zinc-deficient mice is probably related to amelioration of abnormal glucocorticoid secretion. ${ }^{40}$ This is because the basal level of serum glucocorticoid, which was significantly higher in zinc-deficient mice, was decreased by YKS administration, because YKS administration to zincdeficient mice significantly increased serum glucocorticoid levels after a resident-intruder test, and because after the test the significant difference in serum corticosterone levels between control and zinc-deficient mice disappeared. ${ }^{40}$

There are several reports that indicate that the antiaggressive efficacy of YKS is related to the serotonergic system. In one such study, para-chloroamphetamine reduced not only the 5-hydroxytryptamine (5-HT) concentration but also the high potassium-induced 5-HT release in the rat hypothalamus. ${ }^{42}$ In the same study, a single injection of YKS ameliorated the para-chloroamphetamine-induced decrease in social behavior but not aggressive behavior. Additionally, chronic treatment for 14 days with YKS ameliorated parachloroamphetamine-induced abnormal behavior, decreased social behavior, and increased aggressive behavior. The ameliorative effects of chronic YKS on behavior were 
counteracted by the co-administration of WAY-100635, a $5-\mathrm{HT}_{1 \mathrm{~A}}$ antagonist. These results suggest that YKS may have two different effects: an acute effect on social behavior and a chronic effect on aggressive behavior. One of the mechanisms of these effects of YKS may be related to its agonistic effect on $5-\mathrm{HT}_{1 \mathrm{~A}}$ receptors.

Another study suggests that geissoschizine methyl ether in Uncaria hook, a constituent herb of YKS, is a potent $5-\mathrm{HT}_{1 \mathrm{~A}}$ receptor agonist and a candidate for explaining the pharmacological effect of YKS on aggressiveness and sociality in socially isolated mice. ${ }^{44}$

\section{Anxiolytic effects}

Several animal studies have shown anxiolytic effects of YKS. While neither single nor repeated administration of YKS had an effect on basal or stress-induced levels of serum corticosterone, repeated administration of YKS decreased anxiety-like behavior in an open-field test and an elevated plus-maze test in a dose-dependent manner in mice, though a single administration had no effect on locomotor activity or anxiety-like behavior. ${ }^{45}$ In addition, repeated administration of YKS also decreased stress-induced c-Fos expression in the prelimbic cortex and basolateral and medial amygdaloid nuclei. ${ }^{45}$ These results suggest that not single, but repeated, administration of YKS yields anxiolytic effects without affecting serum corticosterone levels, and that its anxiolytic effect may be associated with attenuated neuronal activity in the medial prefrontal cortex and amygdala.

In another study on mice, the anxiolytic effect of YKS was inhibited by pretreatment with the benzodiazepine receptor antagonist flumazenil, suggesting that YKS produces an anxiolytic effect mediated by the benzodiazepine system. ${ }^{46}$ Three months of YKS administration improved aspects of age-related anxiety in F344/N aged rats in a study whose results suggest that this improvement may result from the enhancement of serotonergic and dopaminergic transmissions. ${ }^{47}$

Another rodent behavioral study indicates that the anxiolytic effect of YKS is related to the serotonergic system. ${ }^{48}$ Repeated administration of YKS did not affect the decrease in locomotor activity induced by re-exposure to the footshock box 14 days after footshock stress in an open-field test. In a contextual fear conditioning test, rats that had experienced footshock showed significant freezing behavior on re-exposure to the box 14 days after footshock stress, but YKS significantly suppressed this behavior. In an elevated plus-maze test on the 16th day after the contextual fear conditioning test, YKS significantly increased the time spent in open arms in rats subjected to footshock stress compared to control rats. Interestingly, these anxiolytic effects by YKS were antagonized by WAY-100635, the aforementioned selective 5-HT receptor antagonist, in the contextual fear conditioning test but not in the elevated plus-maze test. Furthermore, the 5-HT receptor agonist buspirone significantly suppressed freezing behavior in the contextual fear conditioning test but induced no change in the time spent in open arms in the elevated plus-maze test. ${ }^{48}$ These findings strongly suggest that YKS has an ameliorating effect on anxiety-like behaviors induced by both innate fear and memory-dependent fear, and that YKS's anxiolytic effect on memory-dependent fear induced by aversive stress is exerted via $5-\mathrm{HT}_{1 \mathrm{~A}}$ receptors.

\section{Efficacy of YKS in dementia models of Alzheimer's disease}

The effects of YKS on dementia have been investigated in rodent models. Three behavioral tests revealed that YKS improves learning and non-cognitive disturbance in Tg2576 mice expressing the human form of APP695SWE (APP-Tg mice), which is considered to be an animal model of Alzheimer's disease. In one study using APP-Tg-positive mice, treatment with YKS for 2 months significantly reduced the increased number of entries and the time spent in open arms in the elevated plus-maze test, treatment for 5 months shortened the time until reaching the platform in the Morris water maze test, and treatment for 9 months significantly suppressed the increase in locomotion in the open-field test. ${ }^{49}$ These results suggest that YKS ameliorates learning deficits and non-cognitive defects including a decrease in anxiety (or disinhibition) and an increase in locomotor activity (hyperactivity) in APP-Tg-positive mice. In another study, the same group reports that YKS inhibited amyloid $\beta$ protein aggregation in a concentration-dependent manner in vitro and that YKS prevented the accumulation of cerebral amyloid $\beta$ protein in vivo using APP-Tg-positive mice, supporting YKS's efficacy in preventing and/or treating Alzheimer's disease. ${ }^{50}$ Additionally, a recent study revealed that YKS has an ameliorative effect on spatial memory impairment in an early-phase Alzheimer's disease rat model, and that its therapeutic mechanism is mediated by an increase in acetylcholine release and the modulation of DMN1 expression, leading to improved synaptic function. ${ }^{51}$ The ameliorative effects of YKS on learning and memory impairments were also studied using olfactory bulbectomized mice, another animal model of Alzheimer's disease. ${ }^{52}$ Olfactory bulbectomized mice caused spatial working memory and non-spatial working memory impairments 
that were reversed by YKS and also by donepezil. In addition, YKS treatment reversed olfactory bulbectomized mice-induced downregulation of choline acetyltransferase and muscarinic $\mathrm{M}_{1}$ receptor expression without affecting muscarinic $\mathrm{M}_{3}$ receptor expression or acetylcholinesterase activity. These results demonstrate that YKS has a therapeutic effect not only on BPSD but also on the memory impairment of Alzheimer's disease that is partly mediated by muscarinic receptor stimulation and the normalization of central cholinergic systems.

\section{Studies using animal models of other dementia types}

There have been several reports using rodent models of dementia other than Alzheimer's disease. Thiamine-deficient (TD) rats, a rodent dementia model, develop degeneration of cerebral cells and dementia-like symptoms, including memory disturbance and behavioral and psychological symptoms. YKS has been found to ameliorate this memory disturbance as well as BPSD-like behavior such as anxiety-like behavior, increases in aggressive behaviors, and decreases in social behaviors in TD rats in a dose-dependent manner. ${ }^{53}$ In addition, the same group showed that YKS ameliorates not only TD-induced aggressive behavior and neurological symptoms but also degeneration of the cerebral cells, which provides further support for YKS's efficacy using electron microscopic evidence. ${ }^{54}$ In another study, the ameliorative effect of YKS on age-related impairments of working memory and reversal learning was examined in aged rats. ${ }^{55}$ The ability to learn initially a reward rule for a T-maze discrimination task (initial learning) was tested in young control (4-month-old), aged control (24-monthold), and YKS-treated aged (24-month-old) rats. Working memory and reversal learning were also examined in delayed alternation and reversal discrimination T-maze tasks, respectively. YKS treatment significantly reversed age-related decreases in accuracy in the delayed alternation and reversal discrimination tasks. These ameliorative effects of YKS on impaired delayed alternation performance were reduced by intracranial infusions of a dopamine D1 receptor antagonist, SCH 23390, into the prelimbic cortical region of the prefrontal cortex, suggesting that YKS's ameliorative effect on age-related impairments of working memory and reversal learning may be mediated by a dopaminergic mechanism in the prefrontal cortex. ${ }^{55}$

There has also been a report on the therapeutic effect of YKS in a rodent model of cerebrovascular dementia. ${ }^{56}$ Rats subjected to repeated cerebral ischemia exhibited anxiety-like behavior in a plus-maze task, a light/dark box test, and an open-field task. Those anxiety-like behaviors were reversed by YKS administration, suggesting that YKS improves BPSD in patients with cerebrovascular dementia as well as other types of dementia.

\section{Animal studies on other clinical efficacies of YKS}

There have been other intriguing animal behavioral studies supporting YKS's other clinical efficacies. YKS has been found to ameliorate vacuous chewing movement in rats, an index for tardive dyskinesia, caused by haloperidol decanoate. ${ }^{57}$ This effect of YKS may be involved in extracellular glutamate level and GLT1(glutamate transporter 1) messenger RNA expression in the striatum. YKS has a reversal effect on the decrease in pentobarbital sleep by social isolation without affecting pentobarbital sleep in group-housed mice. ${ }^{58}$ This prolongation of sleeping time in socially isolated mice is reversed by bicuculline, a competitive antagonist of $\mathrm{GABA}_{\mathrm{A}}$ receptors, and flumazenil, a benzodiazepine receptor antagonist, but not WAY100635, a 5-HT ${ }_{1 \mathrm{~A}}$ receptor antagonist. These results suggest that the $\mathrm{GABA}_{\mathrm{A}}$-benzodiazepine receptor complex, but not $5-\mathrm{HT}_{1 \mathrm{~A}}$ receptors, is involved in YKS's ameliorating effect on sleep.

\section{Potential mechanisms of action of YKS}

The study using the TD rat model revealed that YKS inhibited the degeneration of neuronal and astroglial cells in the brain stem, hippocampus, and cortex. ${ }^{53}$ It also produced a rise in extracellular glutamate in the ventral posterior medial thalamus, suggesting that YKS might inhibit glutamatemediated excitotoxicity. In a separate study by the same research group, YKS was reported to exert neuroprotective effects, protecting against the degeneration of cerebral cells in TD rats. ${ }^{54}$ Neuroprotective effects have also been reported for $\beta$ amyloid-induced cytotoxicity in rat cortical neurons, ${ }^{59}$ glutamate cytotoxicity due to oxidative stress in PC12 cells, ${ }^{60}$ and glutamate-mediated excitotoxicity in cultured cells ${ }^{61}$ and primary cultured rat cortical neurons. ${ }^{62}$ YKS has also been reported to have suppressive effects on excessive glutamate release in zinc-deficient rats. ${ }^{63,64}$ These rodent studies strongly suggest that the glutamatergic neurotransmitter system may play an important role in the mechanism by which YKS exerts a neuroprotective effect.

Another key to YKS's psychopharmacological effects may be the serotonin (5-HT) system. Repeated, but not acute, treatment with YKS $(300 \mathrm{mg} / \mathrm{kg} /$ day for 14 days per os) significantly decreased the expression of $5-\mathrm{HT}_{2 \mathrm{~A}}$ receptors (a receptor subtype in the 5-HT system) in the prefrontal cortex 
and inhibited the head-twitch response in mice induced by 2 , 5-dimethoxy-4-iodoamphetamine (a 5- $\mathrm{HT}_{2 \mathrm{~A}}$ receptor agonist). ${ }^{65}$ As described previously, YKS's anxiolytic effect on memory-dependent fear is exerted via $5-\mathrm{HT}_{1 \mathrm{~A}}$ receptors. ${ }^{48}$ YKS was also found to inhibit the binding of $\left({ }^{3} \mathrm{H}\right) 8-\mathrm{OH}-$ DPAT (a research chemical used to study the function of the $5-\mathrm{HT}_{1 \mathrm{~A}}$ receptor) to $5-\mathrm{HT}_{1 \mathrm{~A}}$ receptors in a concentrationdependent manner. ${ }^{66}$ In another study, the ameliorative effects of YKS on aggressive behavior were counteracted by the coadministration of a $5-\mathrm{HT}_{1 \mathrm{~A}}$ antagonist, ${ }^{42}$ suggesting that YKS has an agonistic effect on $5-\mathrm{HT}_{1 \mathrm{~A}}$ receptors. Given reports that YKS improves abnormalities of the 5-HT system, such as the function of $5-\mathrm{HT}_{2 \mathrm{~A}}$ receptors associated with BPSD in Alzheimer's disease ${ }^{67}$ and reports that tandospirone (a 5- $\mathrm{HT}_{1 \mathrm{~A}}$ partial agonist) improves BPSD symptoms, ${ }^{68}$ the effects of YKS on the serotonin system might play an important role in its clinical effects.

There are several reports on other possible therapeutic mechanisms of YKS. Endoplasmic reticulum stress is known to play an important role in the pathogenesis of Alzheimer's disease, particularly in neuronal death. In one study, YKS inhibited endoplasmic reticulum stressinduced neuronal death in cultured cells by regulating the unfolded protein response. ${ }^{69}$ Another report discusses the effect of YKS on cell signaling pathways. ${ }^{70}$ YKS showed neuroprotective effects in vitro and in vivo and rescued dopaminergic neurons from 1-methyl-4-phenylpyridine/1methyl-4-phenyl-1,2,3,6-tetrahydropyridine toxicity via the $\mathrm{PI} 3 \mathrm{~K} / \mathrm{Akt}$ pathway.

\section{Adverse effects}

The adverse effects of YKS in the human case reports and controlled studies are summarized in Tables 2-4. The most frequently reported side effect is hypokalemia resulting from pseudoaldosteronism, presumably caused by Glycyrrhiza (licorice) root, one of the components of YKS. Potassium supplementation was needed during YKS administration in one case, ${ }^{6}$ and potassium levels quickly returned to baseline levels after patients stopped taking YKS in all cases. Hypokalemia was observed only in trials for dementia, ${ }^{5-7,10,11}$ which might imply that YKS has a greater tendency to cause hypokalemia among the elderly than among the non-elderly.

YKS infrequently evoked tiredness ${ }^{13}$ and oversedation (or drowsiness) 2,3,5,7,35 among the elderly and children and adolescents, and more infrequently caused tiredness in other generations. $^{18,19}$

Gastrointestinal complaints have been reported in the elderly ${ }^{5,11,13}$ and the non-elderly: ${ }^{17-19,33,34}$ however, these complaints are mild and transient. Mild elevation of blood liver enzymes was observed in one case. ${ }^{9}$

There is a concern that YKS might cause interstitial pneumonitis. While this is a very rare adverse effect to Kampo medicines, the potential for interstitial pneumonitis is considered to exist with any Kampo formula. One conference presentation given in Japan and published in abstract form only reported interstitial pneumonitis possibly caused by $\mathrm{YKS},{ }^{71}$ but to the best of the authors' knowledge, there has been no detailed report published to date.

Herbal formulae containing Angelica root or Bupleurum root have been reported to cause hepatotoxic responses in some patients ${ }^{72}$ and YKS should be also monitored for hepatotoxicity and dermatitis caused by a drug-induced autoimmune response.

Given the totality of evidence and the wide use of YKS in the Japanese health care system, this product appears to be very safe for clinical use, with less serious and less frequent adverse effects than the majority of pharmaceutical medications. Nevertheless, when YKS is used, attention should be paid to avoiding and detecting the adverse effects raised in this section.

\section{Conclusion}

YKS appears to have beneficial effects in a variety of diseases, most of which are either psychiatric in nature and/or occur with the aging process. Given the existing evidence and its traditional uses, YKS might be especially useful in clinical conditions accompanied by aggressiveness, impulsive behaviors, and/or involuntary movements. More research is required to further clarify the uses and effectiveness of YKS. In designing future trials, consideration should be given to enrolling participants who also meet the criteria for the use of this formula based on Kampo diagnosis.

In the past decade, YKS became accepted as a standard medicine for BPSD in Japan. It is also officially listed in the Japanese Guidelines for the Management of Dementia as a Grade C1 prescription (recommended regardless of insufficient scientific evidence) for the treatment of sleep disorders and BPSD (especially in DLB) by the Japanese Society of Neurology. This clinical area appears to be a highly promising area for further investigation, and for integration into health care systems outside of Japan. The other areas in which YKS has shown potential for clinical benefit require more clinical and basic studies before any definitive statements can be made about its efficacy and safety. One issue that must be kept in mind is the quality of YKS being used. As mentioned above, Kampo formulas in Japan are standardized and regulated to meet quality control standards akin to those 
for prescription drug medications. Most studies conducted to date have used YKS produced in Japan. This high degree of quality and standardization will not necessarily apply to YKS formulas made outside of Japan.

YKS is not the one and only Kampo formula used widely in the Japanese health care system. Other approved Kampo prescriptions also require additional study to document which might be effective as alternative or accessory treatments for various diseases refractory to conventional medicine as a sole intervention.

\section{Disclosure}

Japanese-Oriental medicine department receives fundings for scholarship activities from Kampo pharmaceutical companies but it is strictly prohibited to use those fundings for research at Chiba University. The authors report no conflicts of interest in this work.

\section{References}

1. Shinno H, Utani E, Okazaki S, et al. Successful treatment with Yi-Gan San for psychosis and sleep disturbance in a patient with dementia with Lewy bodies. Prog Neuropsychopharmacol Biol Psychiatry. 2007;31(7):1543-1545.

2. Iwasaki K, Satoh-Nakagawa T, Maruyama M, et al. A randomized, observer-blind, controlled trial of the traditional Chinese medicine YiGan San for improvement of behavioral and psychological symptoms and activities of daily living in dementia patients. J Clin Psychiatry. 2005;66(2):248-252.

3. Iwasaki K, Maruyama M, Tomita N, et al. Effects of the traditional Chinese herbal medicine Yi-Gan San for cholinesterase inhibitor-resistant visual hallucinations and neuropsychiatric symptoms in patients with dementia with Lewy bodies. J Clin Psychiatry. 2005;66(12): 1612-1613.

4. Shinno H, Inami Y, Inagaki T, Nakamura Y, Horiguchi J. Effect of Yi-Gan San on psychiatric symptoms and sleep structure at patients with behavioral and psychological symptoms of dementia. Prog Neuropsychopharmacol Biol Psychiatry. 2008;32(3):881-885.

5. Mizukami K, Asada T, Kinoshita T, et al. A randomized cross-over study of a traditional Japanese medicine (Kampo), Yokukan-san, in the treatment of the behavioural and psychological symptoms of dementia. Int J Neuropsychopharmacol. 2009;12(2):191-199.

6. Monji A, Takita M, Samejima T, et al. Effect of Yokukan-san on the behavioral and psychological symptoms of dementia in elderly patients with Alzheimer's disease. Prog Neuropsychopharmacol Biol Psychiatry. 2009;33(2):308-311.

7. Hayashi Y, Ishida Y, Inoue T, et al. Treatment of behavioral and psychological symptoms of Alzheimer-type dementia with Yokukan-san in clinical practice. Prog Neuropsychopharmacol Biol Psychiatry. 2010;34(3):541-545.

8. Okahara K, Ishida Y, Hayashi Y, et al. Effects of Yokukan-san on behavioral and psychological symptoms of dementia in regular treatment for Alzheimer's disease. Prog Neuropsychopharmacol Biol Psychiatry. 2010;34(3):532-536.

9. Kawanabe T, Yoritaka A, Shimura H, Oizumi H, Tanaka S, Hattori N. Successful treatment with Yokukan-san for behavioral and psychological symptoms of Parkinsonian dementia. Prog Neuropsychopharmacol Biol Psychiatry. 2010;34(2):284-287.

10. Kimura T, Hayashida H, Furukawa H, Takamatsu J. Pilot study of pharmacological treatment for frontotemporal dementia: effect of Yokukan-san on behavioral symptoms. Psychiatry Clin Neurosci. 2010;64(2):207-210.
11. Iwasaki K, Kosaka K, Mori H, et al. Improvement in delusions and hallucinations in patients with dementia with Lewy bodies upon administration of Yokukan-san, a traditional Japanese medicine. Psychogeriatrics. 2012;12(4):235-241.

12. Nagata K, Yokoyama E, Yamazaki T, et al. Effects of Yokukan-san on behavioral and psychological symptoms of vascular dementia: an open-label trial. Phytomedicine. 2012;19(6):524-528.

13. Sumiyoshi H, Mantani A, Nishiyama S, et al. Yokukan-san treatment of chronic renal failure patients receiving hemodialysis, with behavioral and psychological symptoms of dementia: an open-label study. Am J Geriatr Psychiatry. 2013;21(11):1082-1085.

14. Teranishi M, Kurita M, Nishino S, et al. Efficacy and tolerability of risperidone, Yokukan-san, and fluvoxamine for the treatment of behavioral and psychological symptoms of dementia: a blinded, randomized trial. J Clin Psychopharmacol. 2013;33(5):600-607.

15. Shinno H, Kamei M, Nakamura Y, Inami Y, Horiguchi J. Successful treatment with Yi-Gan San for rapid eye movement sleep behavior disorder. Prog Neuropsychopharmacol Biol Psychiatry. 2008;32(7):1749-1751.

16. Shinno H, Yamanaka M, Ishikawa I, et al. Successful treatment of restless legs syndrome with the herbal prescription Yokukan-san. Prog Neuropsychopharmacol Biol Psychiatry. 2010;34(1):252-253.

17. Miyaoka T, Furuya M, Yasuda H, et al. Yi-Gan San for the treatment of neuroleptic-induced tardive dyskinesia: an open-label study. Prog Neuropsychopharmacol Biol Psychiatry. 2008;32(3):761-764.

18. Miyaoka T, Furuya M, Yasuda H, et al. Yi-Gan San for the treatment of borderline personality disorder: an open-label study. Prog Neuropsychopharmacol Biol Psychiatry. 2008;32(1):150-154.

19. Miyaoka T, Furuya M, Yasuda H, et al. Yi-Gan San as adjunctive therapy for treatment-resistant schizophrenia: an open-label study. Clin Neuropharmacol. 2009;32(1):6-9.

20. Miyaoka T, Wake R, Furuya M, et al. Yokukan-san (TJ-54) for treatment of very-late-onset schizophrenia-like psychosis: an open-label study. Phytomedicine. 2013;20(7):654-658.

21. Okamoto H. Reconsideration of Japanese traditional herbal medicine: new field of research and clinical medicine. Mini Rev Med Chem. 2006;6(5):543-547.

22. Ishibashi A, Kosoto H, Ohno S, Sakaguchi H, Yamada T, and Matsuda K. Introduction to Kampo. Sato Y, Hanawa T, Arai M, et al, editors. Tokyo: Elsevier Japan; 2005:2-13, Chapter 1.

23. Terasawa K. Kampo: Japanese-Oriental Medicine, Insights From Clinical Cases. Tokyo: Standard McIntyre; 1993.

24. Okamoto H, Chino A, Hirasaki Y, Ueda K, Raimura M, Namiki T. A valid approach in refractory glossodynia: a single-institution 5-year experience treating with Japanese traditional herbal (Kampo) medicine. Evid Based Complement Alternat Med. 2013;2013:354872.

25. Yoshikawa M. Textbook of Traditional Japanese Medicine. Part 1: Kampo Medicine. Chapter 5. Tokyo: Health and Labour Sciences; 2013:203.

26. Okamoto H, Okami T, Ikeda M, Takeuchi T. Effects of Yokukan-san on undifferentiated somatoform disorder with tinnitus. Eur Psychiatry. 2005;20(1):74-75.

27. Saito S, Kobayashi T, Osawa T, Kato S. Effectiveness of Japanese herbal medicine Yokukan-san for alleviating psychiatric symptoms after traumatic brain injury. Psychogeriatrics. 2010;10(1): $45-48$.

28. Miyaoka T, Nagahama M, Tsuchie K, et al. Charles Bonnet syndrome: successful treatment of visual hallucinations due to vision loss with Yi-Gan San. Prog Neuropsychopharmacol Biol Psychiatry. 2009;33(2):382-383.

29. Kajitani K, Kanba S. Successful treatment of poststroke emotional incontinence with Yokukan-san, an Asian herbal medicine: report of two cases. J Am Geriatr Soc. 2012;60(2):379-381.

30. Kawabe K, Ueno S, Hosoda Y, Horiguchi J. Nocturnal eating/drinking syndrome with restless legs syndrome caused by neuroleptics improved by Yi-Gan San add-on treatment: a case report. Clin Neuropharmacol. 2012;35(6):290-291. 
31. Okamoto H, Chino A, Hirasaki Y, Ueda K, Iyo M, Namiki T. Orengedoku-to augmentation in cases showing partial response to Yokukan-san treatment: a case report and literature review of the evidence for use of these Kampo herbal formulae. Neuropsychiatr Dis Treat. 2013;9:151-155.

32. Matsuda Y, Kishi T, Shibayama H, Iwata N. Yokukan-san in the treatment of behavioral and psychological symptoms of dementia: a systematic review and meta-analysis of randomized controlled trials. Hum Psychopharmacol. 2013;28(1):80-86.

33. Miyaoka T, Furuya M, Kristian L, et al. Yi-Gan San for treatment of Charles Bonnet syndrome (visual hallucination due to vision loss): an open-label study. Clin Neuropharmacol. 2011;34(1): 24-27.

34. Miyaoka T, Wake R, Furuya M, et al. Yokukan-san (TJ-54) for treatment of pervasive developmental disorder not otherwise specified and Asperger's disorder: a 12-week prospective, open-label study. $B M C$ Psychiatry. 2012;12:215.

35. Wake R, Miyaoka T, Inagaki T, et al. Yokukan-san (TJ-54) for irritability associated with pervasive developmental disorder in children and adolescents: a 12-week prospective, open-label study. J Child Adolesc Psychopharmacol. 2013;23(5):329-336.

36. Satoh T, Takahashi T, Iwasaki K, et al. Traditional Chinese medicine on four patients with Huntington's disease. Mov Disord. 2009;24(3): 453-455.

37. Koshikawa N, Imai T, Takahashi I, Yamauchi M, Sawada S, Kansaku A. Effects of Hochu-ekki-to, Yokukan-san and Saiko-ka-ryukotsu-borei-to on behavioral despair and acetic acid-induced writhing in mice. Methods Find Exp Clin Pharmacol. 1998;20(1):47-51.

38. Suzuki Y, Mitsuhata H, Yuzurihara M, Kase Y. Antiallodynic effect of herbal medicine Yokukan-san on peripheral neuropathy in rats with chronic constriction injury. Evid Based Complement Alternat Med. 2012;2012:953459.

39. Uchida N, Egashira N, Iwasaki K, et al. Yokukan-san inhibits social isolation-induced aggression and methamphetamine-induced hyperlocomotion in rodents. Biol Pharm Bull. 2009;32(3):372-375.

40. Tamano H, Kan F, Oku N, Takeda A. Ameliorative effect of Yokukansan on social isolation-induced aggressive behavior of zinc-deficient young mice. Brain Res Bull. 2010;83(6):351-355.

41. Takeda A, Iwaki H, Ide K, Tamano H, Oku N. Therapeutic effect of Yokukan-san on social isolation-induced aggressive behavior of zincdeficient and pair-fed mice. Brain Res Bull. 2012;87(6):551-555.

42. Kanno H, Sekiguchi K, Yamaguchi T, et al. Effect of Yokukan-san, a traditional Japanese medicine, on social and aggressive behaviour of para-chloroamphetamine-injected rats. J Pharm Pharmacol. 2009;61(9):1249-1256.

43. Sekiguchi K, Yamaguchi T, Tabuchi M, Ikarashi Y, Kase Y. Effects of Yokukan-san, a traditional Japanese medicine, on aggressiveness induced by intracerebroventricular injection of amyloid $\beta$ protein into mice. Phytother Res. 2009;23(8):1175-1181.

44. Nishi A, Yamaguchi T, Sekiguchi K, et al. Geissoschizine methyl ether, an alkaloid in Uncaria hook, is a potent serotonin 1A receptor agonist and candidate for amelioration of aggressiveness and sociality by Yokukan-san. Neuroscience. 2012;207:124-136.

45. Shoji H, Mizoguchi K. Brain region-specific reduction in c-Fos expression associated with an anxiolytic effect of Yokukan-san in rats. J Ethnopharmacol. 2013;149(1):93-102.

46. Kamei J, Miyata S, Ohsawa M. Involvement of the benzodiazepine system in the anxiolytic-like effect of Yokukan-san (Yi-Gan San). Prog Neuropsychopharmacol Biol Psychiatry. 2009;33(8):1431-1437.

47. Mizoguchi K, Tanaka Y, Tabira T. Anxiolytic effect of a herbal medicine, Yokukan-san, in aged rats: involvement of serotonergic and dopaminergic transmissions in the prefrontal cortex. J Ethnopharmacol. 2010;127(1):70-76.

48. Yamaguchi T, Tsujimatsu A, Kumamoto H, et al. Anxiolytic effects of Yokukan-san, a traditional Japanese medicine, via serotonin 5- $\mathrm{HT}_{1 \mathrm{~A}}$ receptors on anxiety-related behaviors in rats experienced aversive stress. J Ethnopharmacol. 2012;143(2):533-539.
49. Tabuchi M, Yamaguchi T, Iizuka S, Imamura S, Ikarashi Y, Kase Y. Ameliorative effects of Yokukan-san, a traditional Japanese medicine, on learning and non-cognitive disturbances in the Tg2576 mouse model of Alzheimer's disease. J Ethnopharmacol. 2009;122(1):157-162.

50. Fujiwara H, Takayama S, Iwasaki K, et al. Yokukan-san, a traditional Japanese medicine, ameliorates memory disturbance and abnormal social interaction with anti-aggregation effect of cerebral amyloid $\beta$ proteins in amyloid precursor protein transgenic mice. Neuroscience. 2011;180:305-313.

51. Uchida N, Takasaki K, Sakata Y, et al. Cholinergic involvement and synaptic dynamin 1 expression in Yokukan-san-mediated improvement of spatial memory in a rat model of early Alzheimer's disease. Phytother Res. 2013;27(7):966-972.

52. Yamada M, Hayashida M, Zhao Q, et al. Ameliorative effects of Yokukan-san on learning and memory deficits in olfactory bulbectomized mice. J Ethnopharmacol. 2011;135(3):737-746.

53. Ikarashi Y, Iizuka S, Imamura S, et al. Effects of Yokukan-san, a traditional Japanese medicine, on memory disturbance and behavioral and psychological symptoms of dementia in thiamine-deficient rats. Biol Pharm Bull. 2009;32(10):1701-1709.

54. Iizuka S, Kawakami Z, Imamura S, et al. Electron-microscopic examination of effects of Yokukan-san, a traditional Japanese medicine, on degeneration of cerebral cells in thiamine-deficient rats. Neuropathology. Epub 2010 Mar 10.

55. Mizoguchi K, Shoji H, Tanaka Y, Tabira T. Ameliorative effect of traditional Japanese medicine Yokukan-san on age-related impairments of working memory and reversal learning in rats. Neuroscience. 2011;177:127-137.

56. Nogami A, Sakata Y, Uchida N, et al. Effects of Yokukan-san on anxiety-like behavior in a rat model of cerebrovascular dementia. J Nat Med. 2011;65(2):275-281.

57. Sekiguchi K, Kanno H, Yamaguchi T, Ikarashi Y, Kase Y. Ameliorative effect of Yokukan-san on vacuous chewing movement in haloperidolinduced rat tardive dyskinesia model and involvement of glutamatergic system. Brain Res Bull. 2012;89(5-6):151-158.

58. Egashira N, Nogami A, Iwasaki K, et al. Yokukan-san enhances pentobarbital-induced sleep in socially isolated mice: possible involvement of $\mathrm{GABA}_{\mathrm{A}}$-benzodiazepine receptor complex. J Pharmacol Sci. 2011;116(3):316-320.

59. Tateno M, Ukai W, Ono T, Saito S, Hashimoto E, Saito T. Neuroprotective effects of Yi-Gan San against $\beta$ amyloid-induced cytotoxicity on rat cortical neurons. Prog Neuropsychopharmacol Biol Psychiatry. 2008;32(7):1704-1707.

60. Kawakami Z, Kanno H, Ikarashi Y, Kase Y. Yokukan-san, a Kampo medicine, protects against glutamate cytotoxicity due to oxidative stress in PC12 cells. J Ethnopharmacol. 2010;134(1):74-81.

61. Kawakami Z, Kanno H, Ueki T, et al. Neuroprotective effects of Yokukan-san, a traditional Japanese medicine, on glutamate-mediated excitotoxicity in cultured cells. Neuroscience. 2009;159(4): 1397-1407.

62. Kawakami Z, Ikarashi Y, Kase Y. Isoliquiritigenin is a novel NMDA receptor antagonist in Kampo medicine Yokukan-san. Cell $\mathrm{Mol} \mathrm{Neu-}$ robiol. 2011;31(8):1203-1212.

63. Takeda A, Itoh H, Tamano H, Yuzurihara M, Oku N. Suppressive effect of Yokukan-san on excessive release of glutamate and aspartate in the hippocampus of zinc-deficient rats. Nutr Neurosci. 2008;11(1): 41-46.

64. Takeda A, Tamano H, Itoh H, Oku N. Attenuation of abnormal glutamate release in zinc deficiency by zinc and Yokukan-san. Neurochem Int. 2008;53(6-8):230-235.

65. Egashira N, Iwasaki K, Ishibashi A, et al. Repeated administration of Yokukan-san inhibits DOI-induced head-twitch response and decreases expression of 5-hydroxytryptamine (5-HT)2A receptors in the prefrontal cortex. Prog Neuropsychopharmacol Biol Psychiatry. 2008;32(6):1516-1520.

66. Terawaki K, Ikarashi Y, Sekiguchi K, Nakai Y, Kase Y. Partial agonistic effect of Yokukan-san on human recombinant serotonin 1A receptors expressed in the membranes of Chinese hamster ovary cells. J Ethnopharmacol. 2010;127(2):306-312. 
67. Pritchard AL, Harris J, Pritchard CW, et al. Role of 5HT 2A and 5HT $2 \mathrm{C}$ polymorphisms in behavioural and psychological symptoms of Alzheimer's disease. Neurobiol Aging. 2008;29(3):341-347.

68. Sato S, Mizukami K, Asada T. A preliminary open-label study of $5-\mathrm{HT}_{1 \mathrm{~A}}$ partial agonist tandospirone for behavioural and psychological symptoms associated with dementia. Int J Neuropsychopharmacol. 2007;10(2):281-283.

69. Hiratsuka T, Matsuzaki S, Miyata S, et al. Yokukan-san inhibits neuronal death during ER stress by regulating the unfolded protein response. PLoS One. 2010;5(10):e13280.
70. Doo AR, Kim SN, Park JY, et al. Neuroprotective effects of an herbal medicine, Yi-Gan San on MPP+/MPTP-induced cytotoxicity in vitro and in vivo. J Ethnopharmacol. 2010;131(2):433-442.

71. Nakamura K, Kawagoe I, Kanai Y, Konishi R, Mitsuhata H. [A case of drug-induced pneumonitis caused by Yokukan-san] [abstract]. Journal of Japan Society of Pain Clinicians. 2010;17(3):384. Abstract P1-2-39. Japanese.

72. Teschke R, Wolff A, Frenzel C, Schulze J, Eickhoff A. Herbal hepatotoxicity: a tabular compilation of reported cases. Liver Int. 2012;32(10): 1543-1556.

\section{Publish your work in this journal}

Neuropsychiatric Disease and Treatment is an international, peerreviewed journal of clinical therapeutics and pharmacology focusing on concise rapid reporting of clinical or pre-clinical studies on a range of neuropsychiatric and neurological disorders. This journal is indexed on PubMed Central, the 'PsycINFO' database and CAS, and is the official journal of The International Neuropsychiatric Association (INA). The manuscript management system is completely online and includes a very quick and fair peer-review system, which is all easy to use. Visit http://www.dovepress.com/testimonials.php to read real quotes from published authors.

Submit your manuscript here: http://www.dovepress.com/neuropsychiatric-disease-and-treatment-journal 